Bentham OPen
CrossMark
Content list available at: www.benthamopen.com/TOMCJ/
DOI: $10.2174 / 1874104501812010074,2018,12,74-83$

\title{
LETTER
}

\section{Discovery of Iminobenzimidazole Derivatives as Novel Cytotoxic Agents}

Nora Chouha ${ }^{1,3}$, Hassan Hammoud ${ }^{1}$, Simone Brogi ${ }^{4}$, Giuseppe Campiani ${ }^{4}$, Caroline Welsch $^{5,6}$, Caroline Robert ${ }^{5,7}$, Stéphan Vagner $^{8}$, Thierry Cresteil ${ }^{9}$, Embarek Bentouhami ${ }^{1,10}$ and Laurent Désaubry ${ }^{1,2, *}$

${ }^{1}$ Laboratory of Therapeutic Innovation (UMR 7200), Faculty of Pharmacy, University of Strasbourg-CNRS, 67401, Illkirch, France

${ }^{2}$ Laboratory of Biomolecules (UMR7203), CNRS-Sorbonne University, 4 place Jussieu, 75005Paris, France

${ }^{3}$ Laboratory of Synthesis of Molecules of Biological Interest, Université des Frères Mentouri Constantine 1, 25000 Constantine, Algeria

${ }^{4}$ European Research Centre for Drug Discovery (Nat SynDrugs), Department of Biotechnology, Chemistry and Pharmacy (DBCF), University of Siena, Siena, Italy

${ }^{5}$ INSERM U981, Gustave Roussy Cancer Campus, Villejuif, France

${ }^{6}$ Université Paris-Sud, Université Paris-Saclay, Kremlin-Bicêtre, France

${ }^{7}$ Department of Oncology, Gustave Roussy Cancer Campus, Villejuif, France

${ }^{8}$ Institut Curie, PSL Research University, CNRS UMR3348,91405, Orsay, France

${ }^{9} I P S I T$, Faculty of Pharmacy, Université Paris-Sud, 92290 Chatenay-Malabry, France

${ }^{10}$ Laboratory of Chemistry, Molecular Engineering and Nanostructures (LCIMN), University Ferhat Abbas- Sétif 1, Sétif, Algeria

\begin{abstract}
In our quest to identify inhibitors of the eukaryotic translation initiation factor 4F (eIF4F), we serendipitously discovered a novel cytotoxic agent. Even though this compound did not inhibit translation, we explored the structural requirements for its cytotoxicity due to its structural originality. A series of 1,3-disubstituted iminobenzimidazoles was synthesized and evaluated for their in vitro cytotoxicity. The structure-activity relationship studies demonstrate that hydrophobic substituent is essential for activity. The most active compounds displayed a cytotoxicity in KB, HL60 and HCT116 human cancer cells with an $\mathrm{IC}_{50}$ of about $1 \mu \mathrm{M}$. These first-in-class series of low molecular weight synthetic molecules may provide the basis for the development of new anticancer drugs.
\end{abstract}

Keywords: Iminobenzimidazoles, Cytotoxicity, Structure-activity relationship, Cancer, Apoptosis, Eukaryotic translation initiation factor $4 \mathrm{~F}$.

\section{INTRODUCTION}

Targeting the eukaryotic translation initiation factor 4F (eIF4F) holds promise as novel anticancer drugs that can overcome intra-tumor heterogeneity [1]. Indeed, the eIF4F complex, which regulates the cap-dependent protein synthesis is dysregulated in many types of cancers, leading to the overexpression of proteins that promote tumor growth, metastasis and resistance to cancer treatments. More important, several natural products that inhibit eIF4F, principally flavaglines, but also hippuristanol and pateamine, have demonstrated promising activities in several mouse

* Address correspondence to this authors at the Faculty of Pharmacy, University of Strasbourg-CNRS, 67401, Illkirch, France; Tel: (+33) 368 854 141; E-mails: desaubry@unistra.fr; labo.strasbg@yahoo.fr 
models of cancers. In our quest to identify novel eIF4F inhibitors [2 - 6], we performed a virtual screening and we identified the iminobenzimidazole 1 (ChemBridge 5657657, Scheme 1). as a putative eIF4F inhibitor. Unfortunately, when we examine whether it inhibits eIF4F by using a bicistronic luciferase reporter construct [3], we didn't find any significant activity. However, we found that it is cytotoxic in A375 melanoma cells. Considering that $\mathbf{1}$ had been described in a small number of patents and articles, none of them were related to cancer [7 - 10], and since the anticancer potential of iminobenzimidazoles has been scarcely investigated [11], we explored the requirement of $\mathbf{1}$ for its cytotoxicity to determine whether it could provide the basis for the development of new anticancer agents.

\section{MATERIALS \& METHODS}

\subsection{Chemical Syntheses}

\subsubsection{2-[(4-Chlorophenoxy) Methyl] Oxirane (3)}

4-Chlorophenol (12.0 g, $93.3 \mathrm{mmol}, 1$ equiv) was taken up into acetone ( $250 \mathrm{~mL})$, then $\mathrm{K}_{2} \mathrm{CO}_{3}(38.7 \mathrm{~g}, 280.0 \mathrm{mmol}$, 3 equiv) and epichlorohydrin $(21.6 \mathrm{~mL}, 280.0 \mathrm{mmol}, 3$ equiv) were added consecutively. The reaction mixture was set to stir at reflux for $24 \mathrm{~h}$. At this time, an additional 3 equiv of epichlorohydrin was added and the solution was allowed to stir at reflux for an additional $24 \mathrm{~h}$. The mixture was cooled down to room temperature, and the solids were filtered off. The solvent was removed under reduced pressure and the resulting oil was taken up in diethyl ether (150 mL). The organic layer was washed with $\mathrm{H}_{2} \mathrm{O}(1 \times 150 \mathrm{~mL}), 1 \mathrm{M}$ aqueous $\mathrm{NaOH}$ solution $(1 \times 150 \mathrm{~mL})$, and brine $(1 \mathrm{x} 150 \mathrm{~mL})$. The organic layer was dried over $\mathrm{MgSO}_{4}$, filtered, and the solvent was removed under reduced pressure. The resulting yellow oil was purified via column $\mathrm{SiO}_{2}$ chromatography (10\% EtOAc/hexanes) to yield the desired epoxide as a yellow oil (15.2 g, 88\%); ${ }^{1} \mathrm{H}$ NMR $\left(\mathrm{CDCl}_{3}, 400 \mathrm{MHz}\right) \delta 7.29-7.25$ (m, 2H), 6.90-6.86 (m, 2H), $4.24(\mathrm{dd}, J=2.8,10.8 \mathrm{~Hz}, 1 \mathrm{H})$, $3.94(\mathrm{dd}, J=2.8,10.8 \mathrm{~Hz}, 1 \mathrm{H}), 3.39-3.35(\mathrm{~m}, 1 \mathrm{H}), 2.94(\mathrm{t}, J=4.4 \mathrm{~Hz}, 1 \mathrm{H}), 2.78(\mathrm{dd}, \mathrm{J}=2.8,4.4 \mathrm{~Hz}, 1 \mathrm{H}) ;{ }^{13} \mathrm{C} \mathrm{NMR}$ $\left(\mathrm{CDCl}_{3}, 100 \mathrm{MHz}\right) \delta 157.1,129.4,126.1,115.9,69.1,50.0,44.6$.

\subsubsection{1-(4-Chlorophenoxy)-3-(2-Imino-2,3-Dihydro-1H-Benzo[d]imidazol-1-yl)Propan-2-ol (4)}

(4-Chlorophenoxy)-3-(2-imino-2,3-dihydro-1H-benzo[d]imidazol-1-yl)propan-2-ol (4). To a solution of 2aminobenzimidazole 2 (4.0 g, $30.04 \mathrm{mmol})$ in $\mathrm{H}_{2} \mathrm{O} /$ Dioxane $35 / 35 \mathrm{~mL}$, KOH (1.6 g, $\left.30.04 \mathrm{mmol}\right)$ and oxirane 3 (5.5 g, $30.04 \mathrm{mmol}$ ) were added. The mixture was stirred at $110{ }^{\circ} \mathrm{C}$ for 2 hours, then cooled to room temperature. Dioxane was removed under reduced pressure and the resulting solution was extracted 3 times with $50 \mathrm{~mL}$ of AcOEt. The organic layer was dried over $\mathrm{MgSO}_{4}$, filtered, and the solvent was removed under reduced pressure. The resulting solid was recrystallized with $\mathrm{EtOH} / \mathrm{H}_{2} \mathrm{O} 3 / 7$ to give the desired product as a white solid $(6.0 \mathrm{~g}, 18.7 \mathrm{mmol}, 63 \%)$. ${ }^{1} \mathrm{H} \mathrm{NMR}$ (DMSO, 400MHz) $\delta 7.34$ (d, $J=8.4 \mathrm{~Hz}, 2 \mathrm{H}), 7.14(\mathrm{t}, J=8.4 \mathrm{~Hz}, 2 \mathrm{H}), 6.90-6.94(\mathrm{~m}, 3 \mathrm{H}), 6.84((\mathrm{t}, J=7.2 \mathrm{~Hz}, 2 \mathrm{H}), 6.26$ $(\mathrm{s}, 2 \mathrm{H}), 5.59$ (s, 1H), 3.93-4.15 (m, 5H). ${ }^{13} \mathrm{C}$ NMR (DMSO, 100MHz) $\delta 157.8,155.8,143.1,135.3,129.7,124.8,120.7$, $118.5,116.7,115.2,108.3,70.4,68.3,45.4$.

\subsubsection{0-[3-(4-Chlorophenoxy)-2-Hydroxypropyl]-3,4-Dihydrobenzo [4,5] Imidazo [1,2-a] Pyrimidin-2(10H)-one (6)}

To a solution of $4(0.1 \mathrm{~g}, 0.31 \mathrm{mmol})$ in acetonitrile $(4 \mathrm{~mL}), 3$-bromo-N,N-dimethylpropanamide $5(0.08 \mathrm{~g}, 0.43$ mmol) was added and the mixture was stirred at reflux for 15 hours. $\mathrm{K}_{2} \mathrm{CO}_{3}(0.63 \mathrm{mmol})$ was added and the mixture was stirred at reflux for 2 days. Water $(10 \mathrm{~mL})$ and AcOEt $(10 \mathrm{~mL})$ were added and the organic layer was extracted, dried over $\mathrm{MgSO}_{4}$ and concentrated. The residue was purified on flash chromatographic using (MeOH/DCM 5/95) to give the desired product as a white solid (0.7 mg, 55\%). 1H NMR (400 MHz, DMSO) $\delta 7.48(\mathrm{~d}, \mathrm{~J}=7.6 \mathrm{~Hz}, 1 \mathrm{H}), 7.38(\mathrm{~d}, \mathrm{~J}=7.6$ $\mathrm{Hz}, 1 \mathrm{H}), 7.32(\mathrm{~d} \mathrm{~J}=8.4 \mathrm{~Hz}, 2 \mathrm{H}), 7.15-7.23(\mathrm{~m}, 2 \mathrm{H}), 6.95(\mathrm{~d}, \mathrm{~J}=8.4 \mathrm{~Hz}, 2 \mathrm{H}), 5.56(\mathrm{~d}, \mathrm{~J}=7.6 \mathrm{~Hz}, 2 \mathrm{H}), 4.25-4.31(\mathrm{~m}$, $1 \mathrm{H}), 4.12-4.21(\mathrm{~m}, 4 \mathrm{H}), 3.93-4.03(\mathrm{~m}, 2 \mathrm{H}), 2.57-2.61(\mathrm{~m}, 2 \mathrm{H}) .13 \mathrm{C} \mathrm{NMR}$ (400 MHz, DMSO) $\delta 175.3,157.7,155.4$, $131.5,130.0,129.6,124.8,122.7,122.5,116.7,110.7,109.2,70.8,67.2,45.3,38.7,29.8 . \mathrm{LCMS}-\mathrm{ESI}(\mathrm{m} / \mathrm{z})[\mathrm{M}+\mathrm{H}]+$ calcd: 372.10 , found 372.02 .

\subsubsection{9-[3-(4-Chlorophenoxy)-2-Hydroxypropyl]-3H-Benzo[d] Imidazo [1,2-a] Imidazol-2(9H)-One (8).}

A mixture of $2(0.1 \mathrm{~g}, 0.31 \mathrm{mmol}, 1$ equiv) was taken up into $4 \mathrm{ml}$ of acetonitrile, then bromoacetyl bromide 7 (0.02 $\mathrm{mL}, 0.31 \mathrm{mmol}, 1$ equiv) and $\mathrm{K}_{2} \mathrm{CO}_{3}(0.08 \mathrm{~g}, 0.62 \mathrm{mmol}, 2$ equiv $)$. The reaction mixture was stirred at reflux for $24 \mathrm{~h}$, the mixture was cooled down to room temperature, and the solid was filtered, to yield the desired product as a white solid (0.126 g, 39\%); ${ }^{1} \mathrm{H}$ NMR (400 MHz, DMSO) $\delta 7.56$ (d, $\left.J=8.0 \mathrm{~Hz}, 1 \mathrm{H}\right), 7.17-7.35$ (m, 5H), 6.955-6.980 (m, 2H), 
$5.55(\mathrm{~d}, J=2.4 \mathrm{~Hz}, 1 \mathrm{H}), 4.43(\mathrm{~s}, 2 \mathrm{H}), 4.17-4.31(\mathrm{~m}, 3 \mathrm{H}), 4.00-4.05(\mathrm{~m}, 2 \mathrm{H}) .{ }^{13} \mathrm{C}$ NMR (400 MHz, DMSO) $\delta 186.1$, $168.5,157.7,133.7,129.7,129.2,124.9,123.4,122.1,116.7,111.7,109.7,70.6,67.1,51.4,46.9$. LCMS-ESI (m/z) [M $+\mathrm{H}]^{+}$calcd: 358.09 , found 358.08 .

\subsubsection{1-(4-Chlorophenoxy)-3-(3-(2-Hydroxyethyl)-2-Imino-2,3-Dihydro-1H-Benzo[d] Imidazol-1-yl) Propan-2-ol (9b).}

A mixture of 4 ( $0.1 \mathrm{~g}, 0.31 \mathrm{mmol}, 1$ equiv) was taken up into $4 \mathrm{ml}$ of acetonitrile then 2-chloroethanol was added ( $0.04 \mathrm{~mL}, 0.62 \mathrm{mmol}, 2.0$ equiv). The reaction mixture was stirred at reflux for $24 \mathrm{~h}$, the mixture was cooled down to room temperature. The solid was filtered, to yield the desired product as a white solid $(0.04 \mathrm{~g}, 32 \%) ;{ }^{1} \mathrm{H} \mathrm{NMR}(400$ MHz, DMSO) $\delta 8.84(\mathrm{bs}, 1 \mathrm{H}), 7.59(\mathrm{~d}, J=9.2 \mathrm{~Hz}, 2 \mathrm{H}), 7.29-7.36(\mathrm{~m}, 4 \mathrm{H}), 7.02(\mathrm{~d}, J=9.2 \mathrm{~Hz}, 2 \mathrm{H}), 5.65(\mathrm{bs}, 1 \mathrm{H}), 5.09$ (bs, 1H), 4.24-4.34 (m, 5H), 4.06-4.09 (m, 2H), $3.74(\mathrm{t}, J=4.8 \mathrm{~Hz}, 2 \mathrm{H}) .{ }^{13} \mathrm{C}$ NMR (DMSO, 100MHz) $\delta 173.0,157.7$, $150.9,130.8,130.7,129.7,124.9,123.6,123.5,116.8,111.1,70.3,67.2,58.9,46.3,45.8$. LCMS-ESI $_{(\mathrm{m} / \mathrm{z})}$ [M+H] $^{+}$ calcd: 362.12 , found 362.05 .

\subsubsection{2-[3-[3-(4-Chlorophenoxy)-2-Hydroxypropyl]-2-Imino-2,3-Dihydro-1H-Benzo[d] Imidazol-1-yl] Acetamide (9b)}

A mixture of 4 ( $0.08 \mathrm{~g}, 0.25 \mathrm{mmol}, 1$ equiv) was taken up into $4 \mathrm{ml}$ of acetonitrile, then chloroacetamide $(0.02 \mathrm{~mL}$, $0.25 \mathrm{mmol}, 1.0$ equiv) was added. The reaction mixture was stirred at reflux for $24 \mathrm{~h}$, the mixture was cooled down to room temperature. The solid was filtered, to yield the desired product as a white solid $(0.03 \mathrm{~g}, 31 \%) ;{ }^{1} \mathrm{H} \mathrm{NMR}(400$ MHz, DMSO) $\delta 8.96(\mathrm{bs}, 2 \mathrm{H}), 7.87(\mathrm{~s}, 1 \mathrm{H}), 7.40-7.53(\mathrm{~m}, 2 \mathrm{H}), 7.29-7.35(\mathrm{~m}, 5 \mathrm{H}), 7.02(\mathrm{~d}, J=7.2 \mathrm{~Hz}, 2 \mathrm{H}), 4.90(\mathrm{~s}$, 2H), 4.29-4.36 (m, 3H), 4.07-4.09 (m, 2H). ${ }^{13} \mathrm{C}$ NMR (400 MHz, DMSO) $\delta 167.1,157.7,151.3,130.7,130.5,129.7$,

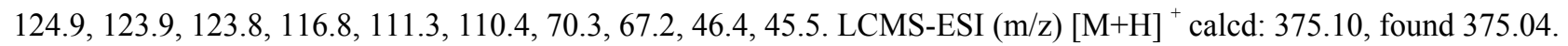

\subsection{7. [3-[2-[1, 3-Dioxolan-2-yl) Ethyl]-2-Imino-2, 3-Dihydro-1H-Benzo[d] Imidazol-1-yl]-3-(4-Chlorophenoxy) Propan-2-ol (9c)}

A mixture of $4(0.2 \mathrm{~g}, 0.63 \mathrm{mmol}, 1$ equiv) was taken up into $4 \mathrm{ml}$ of acetonitrile, then 2-(2-bromoethyl-1,3 dioxane) ( $0.12 \mathrm{~g}, 0.69 \mathrm{mmol}, 1.1$ equiv) was added. The reaction mixture was stirred at reflux for $24 \mathrm{~h}$, the mixture was cooled down to room temperature, and the solid was filtered and purified by column chromatography (AcOEt:MeOH / 9: 1), to yield the desired product as a brown solid (0.1 g, 38\%); ${ }^{1} \mathrm{H}$ NMR (400 MHz, DMSO) $\delta 7.59$ (d, $J=7.6 \mathrm{~Hz}$, $1 \mathrm{H}), 7.51(\mathrm{~d}, J=7.6 \mathrm{~Hz}, 1 \mathrm{H}), 7.28-7.36(\mathrm{~m}, 4 \mathrm{H}), 7.01(\mathrm{~d}, J=8.4 \mathrm{~Hz}, 2 \mathrm{H}), 4.91(\mathrm{t}, J=4.2 \mathrm{~Hz}, 1 \mathrm{H}), 4.23-4.32(\mathrm{~m}, 5 \mathrm{H})$, 4.04-4.1 (m,2H), 3.73,3.88 (m,4H), 2.05 (q, $J=5.7 \mathrm{~Hz}, 2 \mathrm{H}) \cdot{ }^{13} \mathrm{C}$ NMR (400 MHz, DMSO) $\delta 157.7,150.4,130.8$, 129.9, 129.7, 125.0, 123.9, 123.8, LCMS-ESI $(\mathrm{m} / \mathrm{z})[\mathrm{M}+\mathrm{H}]^{+}$calcd: 418.12 , found 418.14 .

\subsubsection{1-(3-Allyl-2-Imino-2,3-Dihydro-1H-Benzo[d] Imidazol-1-yl)-3-(4-Chlorophenoxy)propan-2-ol (9d)}

A mixture of 4 ( $0.12 \mathrm{~g}, 0.40 \mathrm{mmol}, 1$ equiv) was taken up into $4 \mathrm{ml}$ of acetonitrile, then allylbromide $(0.03 \mathrm{~mL}, 0.3$ mmol, 1.0 equiv) was added. The reaction mixture was stirred at reflux for $24 \mathrm{~h}$, the mixture was cooled down to room temperature, and the solid was filtered to yield the desired product as a white solid $(0.05 \mathrm{~g}, 48 \%)$; ${ }^{1} \mathrm{H} \mathrm{NMR}(400 \mathrm{MHz}$, DMSO) $\delta 7.61(\mathrm{dd}, J=7.8 \mathrm{~Hz}, J=2.4 \mathrm{~Hz}, 1 \mathrm{H}), 7.52(\mathrm{dd}, J=7.8 \mathrm{~Hz}, J=2.4 \mathrm{~Hz}, 1 \mathrm{H}), 7.31-7.37(\mathrm{~m}, 4 \mathrm{H}), 7.01(\mathrm{~d}, J=7.8$ $\mathrm{Hz}, 2 \mathrm{H}), 5.83-5.91(\mathrm{~m}, 1 \mathrm{H}), 5.38(\mathrm{~d}, J=7.6 \mathrm{~Hz}, 1 \mathrm{H}), 5.24(\mathrm{~d}, J=7.6 \mathrm{~Hz}, 1 \mathrm{H}), 4.82(\mathrm{~d}, J=3.2 \mathrm{~Hz}, 2 \mathrm{H}), 4.32-4.39$ (m, 2H), 3.92-4.19 (m, 3H). LCMS-ESI $(\mathrm{m} / \mathrm{z})[\mathrm{M}+\mathrm{H}]^{+}$calcd: 357.12 , found 357.13.

\subsubsection{1-(3-Benzyl-2-imino-2,3-Dihydro-1H-Benzo[d] Imidazol-1-yl)-3-(4-Chlorophenoxy)propan-2-ol (9e)}

A mixture of 4 ( $0.09 \mathrm{~g}, 0.30 \mathrm{mmol}, 1$ equiv) was taken up into $4 \mathrm{ml}$ of acetonitrile, then benzylbromide $(0.05 \mathrm{~g}, 0.3$ mmol, 1.0 equiv) was added. The reaction mixture was stirred at reflux for $24 \mathrm{~h}$, the mixture was cooled down to room temperature, and the solid was filtered to yield the desired product as a white solid $(0.06 \mathrm{~g}, 61 \%)$; ${ }^{1} \mathrm{H} \mathrm{NMR}(400 \mathrm{MHz}$, DMSO) $\delta 7.53(\mathrm{~d}, J=7.6 \mathrm{~Hz}, 1 \mathrm{H}), 7.18-7.37(\mathrm{~m}, 10 \mathrm{H}), 7.01(\mathrm{~d}, J=7.6 \mathrm{~Hz}, 2 \mathrm{H}), 5.42(\mathrm{~s}, 2 \mathrm{H}), 4.24-4.28(\mathrm{~m}, 3 \mathrm{H})$, 4.01-4.09 (m, 2H). ${ }^{13} \mathrm{C}$ NMR (DMSO, 100MHz) $\delta 157.7,150.8,135.1,130.8,129.9,129.7,129.3,128.4,127.5,124.9$, 124.1, 124.0, 116.8, 111.6, 111.0, 70.4, 67.0, 46.6, 46.0. LCMS-ESI (m/z) [M+H] ${ }^{+}$calcd: 407.14, found 407.11.

\subsubsection{1-(4-Chlorophenoxy)-3-(2-Imino-3-(3-Phenylpropyl)-2,3-Dihydro-1H-Benzo [d] Imidazol-1-yl) Propan-2-ol (9f)}

A mixture of 4 (0.09 g, $0.30 \mathrm{mmol}, 1$ equiv) was taken up into $4 \mathrm{ml}$ of acetonitrile, then (3-bromopropyl)benzene 
( $0.06 \mathrm{~g}, 0.3 \mathrm{mmol}, 1.0$ equiv) was added. The reaction mixture was stirred at reflux for $24 \mathrm{~h}$, the mixture was cooled down to room temperature, and the solid was filtered to yield the desired product as a white solid $(0.08 \mathrm{~g}, 65 \%)$; ${ }^{1} \mathrm{H}$ NMR (400 MHz, DMSO) $\delta 7.15-7.28(\mathrm{~m}, 10 \mathrm{H}), 7.02(\mathrm{~d}, J=7.6 \mathrm{~Hz}, 1 \mathrm{H}), 6.86(\mathrm{~d}, J=7.6 \mathrm{~Hz}, 2 \mathrm{H}), 4.35-4.71(\mathrm{~m}, 5 \mathrm{H})$, 4.04-4.14 (m, 2H), $2.89(\mathrm{t}, J=7.6 \mathrm{~Hz}, 2 \mathrm{H}), 2.24(\mathrm{t}, J=7.6 \mathrm{~Hz}, 2 \mathrm{H}) .{ }^{13} \mathrm{C}$ NMR (DMSO, 100MHz) $\delta 157.7,150.3,141.3$, 130.7, 130.0, 129.7, 128.8, 128.5, 126.4, 124.9, 123.8, 123.8, 116.8, 111.4, 110.6, 70.4, 67.0, 46.4, 42.9, 32.3, 29.7. LCMS-ESI $(\mathrm{m} / \mathrm{z})[\mathrm{M}+\mathrm{H}]^{+}$calcd: 435.17 , found 435.02 .

\subsubsection{1-(4-Chlorophenoxy)-3-(3-(2-Cyclohexylethyl)-2-Imino-2,3-Dihydro-1H-Benzo[d] Imidazol-1-yl) Propan-2-ol (9g)}

A mixture of 4 ( $0.2 \mathrm{~g}, 0.63 \mathrm{mmol}, 1$ equiv) was taken up into $4 \mathrm{ml}$ of acetonitrile, then 1-bromo-2-cyclohexyl ethane $(0.132 \mathrm{~g}, 0.69 \mathrm{mmol}, 1.1$ equiv) was added. The reaction mixture was stirred at reflux for $24 \mathrm{~h}$, the mixture was cooled down to room temperature, and the solid was filtered, to yield the desired product as a white solid $(0.126 \mathrm{~g}, 39 \%) ;{ }^{1} \mathrm{H}$ NMR (400 MHz, DMSO) $\delta 8.71(\mathrm{sb}, 1 \mathrm{H}), 7.6(\mathrm{~d}, J=8 \mathrm{~Hz}, 1 \mathrm{H}), 7.55(\mathrm{~d}, J=7.2 \mathrm{~Hz}, 1 \mathrm{H}), 7.3-7.36(\mathrm{~m}, 4 \mathrm{H}), 6.99(\mathrm{~d}, J=$ $8.8 \mathrm{~Hz}, 2 \mathrm{H}), 4.08-4.34(\mathrm{~m}, 2 \mathrm{H}), 4.23-4.27(\mathrm{~m}, 1 \mathrm{H}), 4.17(\mathrm{t}, J=7.8 \mathrm{~Hz}, 2 \mathrm{H}), 4.02-4.11(\mathrm{~m}, 2 \mathrm{H}), 1.77(\mathrm{~d}, J=12.4 \mathrm{~Hz}$, $2 \mathrm{H}), 1.63-1.69(\mathrm{~m}, 3 \mathrm{H}), 1.52-1.57(\mathrm{q}, J=7.3 \mathrm{~Hz}, 2 \mathrm{H}), 1.34-1.38(\mathrm{~m}, 1 \mathrm{H}), 1.13-1.25(\mathrm{~m}, 3 \mathrm{H}), 0.93-1.02(\mathrm{~m}, 2 \mathrm{H}) ;{ }^{13} \mathrm{C}$ NMR (400 MHz, DMSO) $\delta$ 156.6, 148.1, 128.6, 127.6, 122.9, 121.8, 121.7, 114.7, 109.4, 108.5, 68.3, 64.9, 44.3, 39.2, 33.0, 32.9, 30.9, 24.3, 24.0 . LCMS-ESI $(\mathrm{m} / \mathrm{z})[\mathrm{M}+\mathrm{H}]^{+}$calcd: 428.12, found 428.18 .

\subsubsection{2. (E)-1-(4-Chlorophenoxy)-3-(2-Imino-3-(3-Phenylallyl)-2,3-Dihydro-1H-Benzo[d] Imidazol-1-yl)-Propan-2-ol (9h)}

A mixture of 4 ( $0.2 \mathrm{~g}, 0.63 \mathrm{mmol}, 1$ equiv) was taken up into $4 \mathrm{ml}$ of acetonitrile, then 3-bromo-1phenyl-1-propene $(0.137 \mathrm{~g}, 0.69 \mathrm{mmol}, 1.1$ equiv) $)$ was added. The reaction mixture was stirred at reflux for $24 \mathrm{~h}$, the mixture was cooled down to room temperature, and the solid was filtered, to yield the desired product as a white solid $(0.186 \mathrm{~g}, 58 \%) ;{ }^{1} \mathrm{H}$ NMR (400 MHz, DMSO) $\delta 7.51(\mathrm{t}, J=3.2, \mathrm{~Hz}, 2 \mathrm{H}), 7.42(\mathrm{~d}, 7.2 \mathrm{~Hz}, 2 \mathrm{H}), 7.25-7.35(\mathrm{~m}, 7 \mathrm{H}), 7.0(\mathrm{~d}, J=8.8 \mathrm{~Hz}, 2 \mathrm{H})$, $6.7(\mathrm{~d}, J=16 \mathrm{~Hz}, 1 \mathrm{H}), 6.39(\mathrm{dt}, J=15.6,5.7 \mathrm{~Hz}, 1 \mathrm{H}), 5.0(\mathrm{~d}, J=5.2,2 \mathrm{H}), 4.27-4.33(\mathrm{~m}, 3 \mathrm{H}), 4.04-4.12(\mathrm{~m}, 2 \mathrm{H}),{ }^{13} \mathrm{C}$ NMR (400 MHz, DMSO) $\delta 157.8,150.7,136.2,136.1,133.1,131.7,130.9,130.1,129.8,129.2,128.6,126.9,124.9$, 123.8, 122.6, 116.8, 111.2, 110.7, 70.5, 67.1, 46.4, 44.6. LCMS-ESI (m/z) $[\mathrm{M}+\mathrm{H}]^{+}$calcd: 434.1, found 434.1.

\subsubsection{1-(4-Chlorophenoxy)-3-(2-Imino-3-(3-Phenylprop-2-yn-1-yl)-2,3-Dihydro-1H-Benzo [d] Imidazol-1-yl) Propan-2-ol (9i)}

A mixture of 4 ( $0.15 \mathrm{~g}, 0.47 \mathrm{mmol}, 1$ equiv) was taken up into $4 \mathrm{ml}$ of acetonitrile, then 3-chloro-1 phenyl-1-propyne $(0.077 \mathrm{~g}, 0.51 \mathrm{mmol}, 1.1$ equiv) was added. The reaction mixture was stirred at reflux for $24 \mathrm{~h}$ and cooled down to room temperature. The solid was filtered to yield the desired product as a white solid $(0.143 \mathrm{~g}, 66 \%) ;{ }^{1} \mathrm{H} \mathrm{NMR}(400 \mathrm{MHz}$, DMSO) $\delta 7.7(\mathrm{~d}, J=7.6 \mathrm{~Hz}, 1 \mathrm{H}), 7.62(\mathrm{~d}, J=8,1 \mathrm{H}), 7.31-7.44(\mathrm{~m}, 9 \mathrm{H}), 7.0(\mathrm{~d}, J=9.6,2 \mathrm{H}), 5.5(\mathrm{~s}, 2 \mathrm{H}), 4.33-4.42$ $(\mathrm{m}, 2 \mathrm{H}), 4.23-4.27(\mathrm{~m}, 1 \mathrm{H}), 4.06-4.1(\mathrm{~m}, 2 \mathrm{H}),{ }^{13} \mathrm{C}$ NMR (400 MHz, DMSO) $\delta 157.7,150.3,132.1,130.9,129.8,129.7$, $129.5,129.3,124.9,124.2,124.1,121.6,116.9,116.8,111.6,110.9,85.3,82.6,70.4,67.1,46.6$, 33.9 . LCMS-ESI $(\mathrm{m} / \mathrm{z})[\mathrm{M}+\mathrm{H}]^{+}$calcd: 432.1, found 432.1.

\subsubsection{1-(4-Chlorophenoxy)-3-(2-Imino-3-(2-Phenoxyethyl)-2,3-Dihydro-1H-Benzo[d] Imidazol-1-yl) Propan-2-ol} (9j)

A mixture of 4 ( $0.2 \mathrm{~g}, 0.63 \mathrm{mmol}, 1$ equiv) was taken up into $4 \mathrm{ml}$ of acetonitrile, then 2-phenoxyethyl bromide $(0.138 \mathrm{~g}, 0.69 \mathrm{mmol}, 1.1$ equiv) was added. The reaction mixture was stirred at reflux for $24 \mathrm{~h}$ and cooled down to room temperature. The solid was filtered to yield the desired product as a white solid $(0.27 \mathrm{~g}, 85 \%) ;{ }^{1} \mathrm{H} \mathrm{NMR}(400 \mathrm{MHz}$, DMSO) $\delta 7.63(\mathrm{~d}, J=7.6 \mathrm{~Hz}, 1 \mathrm{H}), 7.55(\mathrm{~d}, J=7.6 \mathrm{~Hz}, 1 \mathrm{H}), 7.23-7.33(\mathrm{~m}, 6 \mathrm{H}), 6.99(\mathrm{~d}, J=8.8,2 \mathrm{H}), 6.92(\mathrm{t}, J=7.4 \mathrm{~Hz}$, $1 \mathrm{H}), 6.82(\mathrm{~d}, J=8 \mathrm{~Hz}, 2 \mathrm{H}), 4.6(\mathrm{~m}, 2 \mathrm{H}), 4.23-4.32(\mathrm{~m}, 5 \mathrm{H}), 4.02-4.1(\mathrm{~m}, 2 \mathrm{H}),{ }^{13} \mathrm{C}$ NMR $(400 \mathrm{MHz}, \mathrm{DMSO}) \delta 158.3$, $157.7,151.1,130.8,130.6,130.2,130,129.7,124.9,123.6,121.5,116.8,114.8,111.2,111.1,111,70.4,67.1,65.6$, 46.4, 42.9. LCMS-ESI (m/z) $[\mathrm{M}+\mathrm{H}]^{+}$calcd: 438.11, found 438.21.

\subsubsection{1-(3-(4-Bromophenethyl)-2-Imino-2,3-Dihydro-1H-Benzo[d] Imidazol-1-yl)-3-(4-Chlorophenoxy) Propan-2- ol $(9 k)$}

A mixture of $4(0.2 \mathrm{~g}, 0.63 \mathrm{mmol}, 1$ equiv) was taken up into $4 \mathrm{ml}$ of acetonitrile, then 1-bromoethyl - 4-(2bromoethylbenzene) $(0.18 \mathrm{~g}, 0.69 \mathrm{mmol}, 1.1$ equiv) was added. The reaction mixture was stirred at reflux for $24 \mathrm{~h}$ and 
cooled down to room temperature. The solid was filtered to yield the desired product as a white solid $(0.144 \mathrm{~g}, 46 \%) ;{ }^{1} \mathrm{H}$ NMR (400 MHz, DMSO) $\delta$ 7.53-7.55 (m, 1H), $7.47(\mathrm{~d}, J=7.6 \mathrm{~Hz}, 3 \mathrm{H}), 7.35(\mathrm{~d}, J=8.8 \mathrm{~Hz}, 2 \mathrm{H}), 7.27(\mathrm{~d}, J=7.6 \mathrm{~Hz}$, $4 \mathrm{H}), 7.0(\mathrm{~d}, J=8.8 \mathrm{~Hz}, 2 \mathrm{H}), 4.38(\mathrm{t}, J=7.4,2 \mathrm{H}), 4.21-4.29(\mathrm{~m}, 3 \mathrm{H}), 4.02-4.08(\mathrm{~m}, 2 \mathrm{H}), 4.97(\mathrm{t}, J=7.4 \mathrm{~Hz}, 2 \mathrm{H}) .{ }^{13} \mathrm{C}$ NMR (400 MHz, DMSO) 157.7, 152.6, 150.6, 137.2, 131.9, 131.6, 130.7, 129.9, 129.8, 124.9, 123.6, 120.4, 116.8, 111.0, 110.5, 70.4, 67.2, 46.2, 43.7, 31.9. LCMS-ESI $(\mathrm{m} / \mathrm{z})[\mathrm{M}+\mathrm{H}]^{+}$calcd: 499.1, found 499.9.

\subsubsection{1-(4-Chlorophenoxy)-3-[2-Imino-3-[2-(Naphthalen-1-yl) Ethyl]-2, 3-Dihydro-1H-Benzo[d] Imidazol-1-yl] Propan-2-ol (9l)}

A mixture of 4 ( $0.2 \mathrm{~g}, 0.63 \mathrm{mmol}, 1$ equiv) was taken up into $4 \mathrm{ml}$ of acetonitrile, then 1-(2-bromoethyl) naphthalene $(0.16 \mathrm{~g}, 0.69 \mathrm{mmol}, 1.1$ equiv) was added. The reaction mixture was stirred at reflux for $24 \mathrm{~h}$ and cooled down to room temperature. The solid was filtered to yield the desired product as a white solid $(0.281 \mathrm{~g}, 88 \%) ;{ }^{1} \mathrm{H} \mathrm{NMR}(400 \mathrm{MHz}$, DMSO) $\delta 8.08(\mathrm{~s}, 2 \mathrm{H}), 8.06(\mathrm{~d}, J=8.4 \mathrm{~Hz}, 1 \mathrm{H}), 7.9(\mathrm{~d}, J=7.6 \mathrm{~Hz}, 1 \mathrm{H}), 7.77-7.79(\mathrm{~m}, 1 \mathrm{H}), 7.5-7.58(\mathrm{~m}, 3 \mathrm{H}), 7.35-7.38$ $(\mathrm{m}, 4 \mathrm{H}), 7.21(\mathrm{t}, J=7.4 \mathrm{~Hz}, 1 \mathrm{H}), 7.08-7.132(\mathrm{~m}, 2 \mathrm{H}), 7.00-7.06(\mathrm{~m}, 2 \mathrm{H}), 5.63(\mathrm{~s}, 1 \mathrm{H}), 4.59(\mathrm{t}, J=6.6 \mathrm{~Hz}, 2 \mathrm{H})$, 4.23-4.31 (m, 3H), 4.00-4.09 (m, 2H), $3.5(\mathrm{t}, J=6.8 \mathrm{~Hz}, 2 \mathrm{H}) .{ }^{13} \mathrm{C}$ NMR (400 MHz, DMSO) $\delta 157.7,150.3,133.9$, $133.8,131.9,130.4,129.9,129.8,129.1,127.8,127.5,126.7,126.2,125.9,125.0,123.7,116.8,111.3,110.4,70.4$, 67.1, 46.3, 43.7, 30.2. LCMS-ESI (m/z) $[\mathrm{M}+\mathrm{H}]^{+}$calcd: 472.2 , found 472.1 .

\subsubsection{1-(4-Chlorophenoxy)-3-(3-(3,3-Diphenylpropyl)-2-Imino-2,3-Dihydro-1H-Benzo [d] Imidazol-1-yl) Propan-2- ol (9m)}

A mixture of 4 ( $0.2 \mathrm{~g}, 0.63 \mathrm{mmol}, 1$ equiv) was taken up into $4 \mathrm{ml}$ of acetonitrile, then 3,3-diphenyl-1-bromopropane $(0.18 \mathrm{~g}, 0.69 \mathrm{mmol}, 1.1$ equiv) was added. The reaction mixture was stirred at reflux for $24 \mathrm{~h}$ and cooled down to room temperature. The solid was filtered to yield the desired product as a white solid $(0.37 \mathrm{~g}, 86 \%) ;{ }^{1} \mathrm{H} \mathrm{NMR}(400 \mathrm{MHz}$, DMSO) $\delta 8.74(\mathrm{~s}, 2 \mathrm{H}), 7.52-7.55(\mathrm{~m}, 1 \mathrm{H}), 7.26-7.35(\mathrm{~m}, 12 \mathrm{H}), 7.18(\mathrm{t}, J=6.8 \mathrm{~Hz}, 2 \mathrm{H}), 7.10-7.16(\mathrm{~m}, 1 \mathrm{H}), 7.01(\mathrm{~d}, J=$ $8.8 \mathrm{~Hz}, 2 \mathrm{H}), 5.59(\mathrm{~s}, 1 \mathrm{H}), 4.21(\mathrm{~s}, 3 \mathrm{H}), 4.02-4.14(\mathrm{~m}, 5 \mathrm{H}), 5.53-5.55(\mathrm{~m}, 2 \mathrm{H}) .{ }^{13} \mathrm{C}$ NMR $(400 \mathrm{MHz}, \mathrm{DMSO}) \delta 157.7$, $150.1,144.4,144.3,130.6,129.7,129.0,128.0,127.8,126.8,124.9,123.8,116.8,111.4,110.2,70.4,67.0,48.4,46.3$, 42.1, 32.7. LCMS-ESI $(\mathrm{m} / \mathrm{z})[\mathrm{M}+\mathrm{H}]^{+}$calcd: 512.2 , found 512.11 .

\subsubsection{N-Methyl-3-Nitropyridin-2-Amine (11)}

To a solution of methylamine $(10 \mathrm{~mL}, 80.7 \mathrm{mmol})$ at $0^{\circ} \mathrm{C}, 2$-chloro-3-nitropyridine 10 (1.6 g, $\left.10.0 \mathrm{mmol}\right)$ was added in several portions. The solution was stirred 1 hour at $0^{\circ} \mathrm{C}$ then 2 hours at room temperature before concentration in vacuum. The residue was washed with water $(20 \mathrm{~mL})$ and then extracted 3 times in AcOEt $(20 \mathrm{~mL})$. The organic layer was dried over $\mathrm{MgSO}_{4}$ then concentrated to give the desired product as a yellow oil (1.3 g, 85\%). ${ }^{1} \mathrm{H}$ NMR (400 $\left.\mathrm{MHz}, \mathrm{CDCl}_{3}\right) \delta 8.40-8.45(\mathrm{~m}, 2 \mathrm{H}), 8.24(\mathrm{bs}, 1 \mathrm{H}), 6.62-6.65(\mathrm{~m}, 1 \mathrm{H}), 3.16(\mathrm{~d}, J=2.4 \mathrm{~Hz}, 3 \mathrm{H}) .{ }^{13} \mathrm{C}$ NMR $(400 \mathrm{MHz}$, $\left.\mathrm{CDCl}_{3}\right) \delta 155.7,153.2,135.2,128.3,111.5,28.1$.

\subsubsection{N-Methylpyridine-2,3-Diamine (12)}

To a solution of $11(1.1 \mathrm{~g}, 7.18 \mathrm{mmol})$ in dry $\mathrm{MeOH}(25 \mathrm{~mL})$, palladium $10 \%$ carbon $(0.07 \mathrm{~g}, 0.71 \mathrm{mmol})$ was added. The mixture was stirred at room temperature and under hydrogen (atmospheric pressure) overnight. The solution was filtered over celite then concentrated to give the desired product as a yellow oil $(0.88 \mathrm{~g}, 99 \%) .{ }^{1} \mathrm{H} \mathrm{NMR}(400 \mathrm{MHz}$, $\left.\mathrm{CDCl}_{3}\right) \delta 7.78(\mathrm{~d}, J=5.2 \mathrm{~Hz}, 1 \mathrm{H}), 6.83(\mathrm{~d}, J=5.2 \mathrm{~Hz}, 1 \mathrm{H}), 6.51(\mathrm{t}, J=5.4 \mathrm{~Hz}, 1 \mathrm{H}) .4 .20(\mathrm{bs}, 1 \mathrm{H}), 3.18(\mathrm{bs}, 2 \mathrm{H}), 3.00$ $(\mathrm{d}, J=2.4 \mathrm{~Hz}, 3 \mathrm{H}) .{ }^{13} \mathrm{C} \mathrm{NMR}\left(400 \mathrm{MHz}, \mathrm{CDCl}_{3}\right) \delta 151.1,139.2,128.5,121.7,113.2,28.7$.

\subsubsection{3-Methyl-3H-Imidazo[4,5-b] Pyridin-2-Amine (13)}

To solution of $12(0.6 \mathrm{~g}, 4.87 \mathrm{mmol})$ in $\mathrm{MeOH} / \mathrm{H}_{2} \mathrm{O} 12 / 12 \mathrm{~mL}$, cyanogen bromide $(1.5 \mathrm{~g}, 14.63 \mathrm{mmol})$ was added. The mixture was stirred at $60^{\circ} \mathrm{C}$ for 4 hours. After cooling to room temperature, $\mathrm{MeOH}$ was evaporated and the solution was basified to $\mathrm{pH} 8$ using a solution of $\mathrm{NaOH} 1 \mathrm{~N}$. The organic layer was extracted 3 times with AcOEt $(20 \mathrm{~mL})$, dried over $\mathrm{MgSO} 4$ and concentrated in vacuum. The residue was purified by flash chromatographic (MeOH/DCM 1/9) to give the desired product as a brown solid $(0.37 \mathrm{~g}, 55 \%) .{ }^{1} \mathrm{H}$ NMR $(400 \mathrm{MHz}, \mathrm{DMSO}) \delta 7.84(\mathrm{~d}, J=7.2 \mathrm{~Hz}, 1 \mathrm{H}), 7.39$ $(\mathrm{dd}, J=7.2 \mathrm{~Hz}, J=1.8 \mathrm{~Hz}, 1 \mathrm{H}), 6.92-6.96(\mathrm{~m}, 1 \mathrm{H}), 6.78(\mathrm{~s}, 2 \mathrm{H}) .3 .51(\mathrm{~s}, 3 \mathrm{H}) .{ }^{13} \mathrm{C}$ NMR $(400 \mathrm{MHz}, \mathrm{DMSO}) \delta 156.5$, $148.5,137.8,136.3,120.5,117.1,27.3$. 
2.1.21. 2.1.21 1-(4-Chlorophenoxy)-3-(2-Imino-3-Methyl-2,3-Dihydro-1H-Imidazo[4,5-b] Pyridin-1-yl) Propan-2-ol (14)

To a solution of 3-methyl-3H-imidazo [4,5-b]pyridin-2-amine $(0.1 \mathrm{~g}, 0.67 \mathrm{mmol})$ in $\mathrm{H}_{2} \mathrm{O} /$ dioxane $3 / 3 \mathrm{~mL}, \mathrm{KOH}$ ( $0.04 \mathrm{~g}, 0.67 \mathrm{mmol})$ and epoxide $3(0.13 \mathrm{~g}, 0.74 \mathrm{mmol})$ were added. The mixture was stirred at $110^{\circ} \mathrm{C}$ for 2 hours, then cooled down to room temperature. Dioxane was removed under reduced pressure and the resulting solution was extracted 3 times with $50 \mathrm{~mL}$ of AcOEt. The organic layer was dried over $\mathrm{MgSO}_{4}$, filtered, and the solvent was removed under reduced pressure. The resulting solid was recrystallized with $\mathrm{EtOH} / \mathrm{H}_{2} \mathrm{O} 3 / 7$ to give the desired product as a white solid $(0.05 \mathrm{~g}, 0.16 \mathrm{mmol}, 25 \%) .{ }^{1} \mathrm{H} \mathrm{NMR}\left(\mathrm{CDCl}_{3}, 400 \mathrm{MHz}\right) \delta 8.11(\mathrm{~d}, J=4.4 \mathrm{~Hz}, 1 \mathrm{H}), 7.65(\mathrm{~d}, J=7.6 \mathrm{~Hz}$, $1 \mathrm{H}), 7.28$ (d, $J=7.6 \mathrm{~Hz}, 1 \mathrm{H}), 6.87$ (d, $J=7.6 \mathrm{~Hz}, 1 \mathrm{H}), 5.08$ (bs, 1H), 4.31-4.42 (m, 1H), 3.94-4.09 (m, 3H), 3.76-3.80 $(\mathrm{m}, 1 \mathrm{H}), 3.64(\mathrm{~s}, 3 \mathrm{H}) .{ }^{13} \mathrm{C} \mathrm{NMR}\left(400 \mathrm{MHz}, \mathrm{CDCl}_{3}\right) \delta 157.1,155.4,147.9,139.7,134.2,129.4,126.1,122.4,117.7$, 115.7, 70.1, 69.4, 46.5, 26.8. LCMS-ESI (m/z) $[\mathrm{M}+\mathrm{H}]^{+}$calcd: 333.10 , found 333.04.

\subsection{Biological Assays}

\subsubsection{Cell Culture}

The human cell lines KB (nasopharyngeal epidermis carcinoma) and HCT116 (colon adenocarcinoma) were purchased from ECACC (Salisbury, UK) and HL60 (promyeocytic leukaemia) cells from ATCC. KB cells were grown in D-MEM medium supplemented with $10 \%$ fetal calf serum, in the presence of penicillin, streptomycin and fungizone in a $75 \mathrm{~cm}^{2}$ flask under $5 \% \mathrm{CO}^{2}$, whereas the two other cell lines were grown in complete RPMI medium.

\subsubsection{Cell Cytotoxicity Assay}

Cells were plated in 96-well tissue culture plates in $200 \mu \mathrm{l}$ medium and treated $24 \mathrm{~h}$ later with complexes dissolved in DMSO at concentrations ranged $0.5 \mathrm{nM}$ to $10 \mu \mathrm{M}$ and were prepared by using a Biomek 3000 (Beckman-Coulter). Control cells received the same volume of DMSO (1\% final volume). After $72 \mathrm{~h}$ exposure to the drug, Cell Titer $96^{\circledR}$ AQuerous one reagent (Promega) was added and incubated for $3 \mathrm{~h}$ at $37{ }^{\circ} \mathrm{C}$ : the absorbance was monitored at 490 $\mathrm{nm}$ and results are expressed as the inhibition of cell proliferation and cell viability calculated as the ratio [(1-(OD490 treated/OD490 control) $) \times 100]$. For $\mathrm{IC}_{50}$ determinations $(50 \%$ inhibition of cell viability), experiments were performed in duplicate.

\section{RESULTS AND DISCUSSION}

We started our Structure-Activity Relationships (SAR) investigation by replacing the $N$-methyl of 1 by various substituents. The synthesis of these derivatives began with the alkylation of aminobenzimidazole 2 with an epoxide 3 to afford adduct 4 Scheme (1). Condensation of $\mathbf{4}$ with acyl halides 5 and $\mathbf{7}$ delivered the cyclized adducts 6 and 8. Reaction with alkyl halides afforded adducts 9a-p (Scheme 1).

Aza-isostere 14 was prepared in 4 steps starting from 2-chloro-3-nitropyridine 10 (Scheme 2). Condensation with methylamine, followed by a reduction of the nitro group and ring closure with cyanogen bromide afforded the azabenzimidazole 13 that was alkylated by epoxide 3 to yield 14 .

Surprisingly, neither 1 nor its desmethyl analogue $\mathbf{4}$ was cytotoxic to KB cells at $10 \mu \mathrm{M}$ (Table 1). The inclusion of the amidine moiety in an extra cycle $(\mathbf{6}, \mathbf{8})$ or introduction of polar functions $(\mathbf{9 a - c )}$ did not promote cytotoxicity. Introduction of a nitrogen in the benzimidazole moiety (14) was also ineffective, but the replacement of the methyl by an allyl (9d) or benzyl (9e) made these compounds extremely cytotoxic. Gratefully, increasing the size of the linker between iminobenzimidazolium and phenyl from one to three methylenes $(\mathbf{9 f})$ enhanced cytotoxicity. 
<smiles></smiles>

Scheme (1). Structure of hit $\mathbf{1}$ and synthesis of its analogues $\mathbf{6 , 8}$ and $\mathbf{9 a - p .}$

Aza-isostere 14 was prepared in 4 steps starting from 2-chloro-3-nitropyridine 10 Scheme. (2). Condensation with methylamine, followed by a reduction of the nitro group and ring closure with cyanogen bromide afforded the azabenzimidazole 13 that was alkylated by epoxide 3 to yield 14. Synthesis of aza-isostere 14.

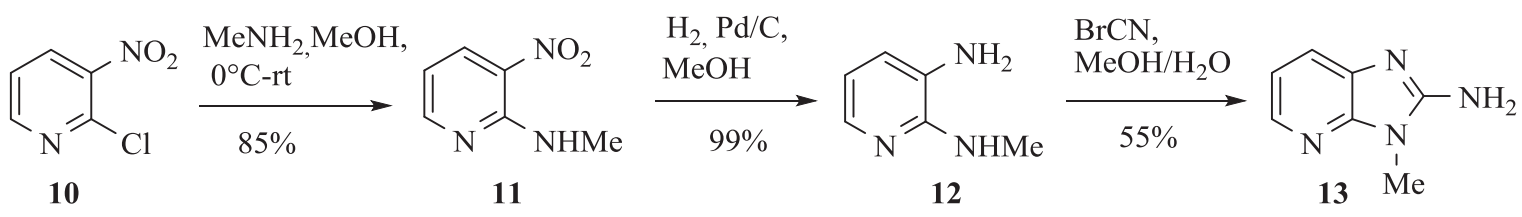<smiles>Cn1c(=N)n(CC(O)COc2ccc(Cl)cc2)c2ccc(O)nc21</smiles>

Scheme (2). Synthesis of aza-isostere 14.

With the identification of potent cytotoxic compounds, we pursued our investigation by determining the $\mathrm{IC}_{50}$ of newly synthesized compounds in 3 human cancer cell lines (Table 2), (Fig. 1). These three human cancer cell lines are respectively derived from patients with cervical carcinoma, acute promyelocytic leukemia and colorectal carcinoma. As such, they represent a variety of different cancers. Replacement of the phenyl by a cyclohexyl $(\mathbf{9 g})$, introduction of unsaturations or an ether in the linker $(\mathbf{9 h}-\mathbf{j})$, and the addition of a bromine (9k) or extra aromatic cycles (91,m) did not significantly modified cytotoxicity, which was very similar in the 3 examined cell lines. These results suggest that these compounds probably bind with a comparable affinity for their target and indicate the requirement for a hydrophobic moiety connected to the benzimidazole ring by a linker with 2 or 3 carbons (Fig. 2). 
Table 1. Cytotoxicity in KB, HL60 and HCT116 human cancer cells $\left(\mathrm{IC}_{50}, \mu \mathrm{M}\right){ }^{\mid \text {a] }}$<smiles>[X][M]n1c(=N)n(CC(O)COc2ccc(Cl)cc2)c2ccccc21</smiles>

\begin{tabular}{|c|c|c|c|c|c|c|}
\hline Cpd. & $\mathbf{R}$ & $\mathbf{R}^{\prime}$ & $\mathbf{A}$ & HX & $10^{-5} M$ & $10^{-6} M$ \\
\hline 1 & $\mathrm{Me}$ & $\mathrm{H}$ & $\mathrm{CH}$ & - & $0 \pm 17$ & $0 \pm 2$ \\
\hline 4 & $\mathrm{H}$ & $\mathrm{H}$ & $\mathrm{CH}$ & - & $0 \pm 9$ & $0 \pm 3$ \\
\hline 6 & & & $\mathrm{CH}$ & - & $0 \pm 3$ & $0 \pm 2$ \\
\hline 8 & & & $\mathrm{CH}$ & - & $0 \pm 6$ & $0 \pm 7$ \\
\hline $9 a$ & & $\mathrm{H}$ & $\mathrm{CH}$ & $\mathrm{HCl}$ & $0 \pm 12$ & $0 \pm 15$ \\
\hline $9 \mathrm{~b}$ & & $\mathrm{H}$ & $\mathrm{CH}$ & $\mathrm{HCl}$ & $0 \pm 2$ & $0 \pm 9$ \\
\hline $9 \mathrm{c}$ & & $\mathrm{H}$ & $\mathrm{CH}$ & - & $1 \pm 11$ & $0 \pm 13$ \\
\hline 14 & $\mathrm{Me}$ & $\mathrm{H}$ & $\mathrm{N}$ & - & $0 \pm 7$ & $0 \pm 4$ \\
\hline 9d & & $\mathrm{H}$ & $\mathrm{CH}$ & $\mathrm{HBr}$ & $88 \pm 5$ & $0 \pm 17$ \\
\hline $9 \mathrm{e}$ & & $\mathrm{H}$ & $\mathrm{CH}$ & $\mathrm{HBr}$ & $96 \pm 2$ & $8 \pm 8$ \\
\hline 9f & & $\mathrm{H}$ & $\mathrm{CH}$ & $\mathrm{HBr}$ & $98 \pm 1$ & $57 \pm 5$ \\
\hline
\end{tabular}

[a] Experiments were performed in triplicate.
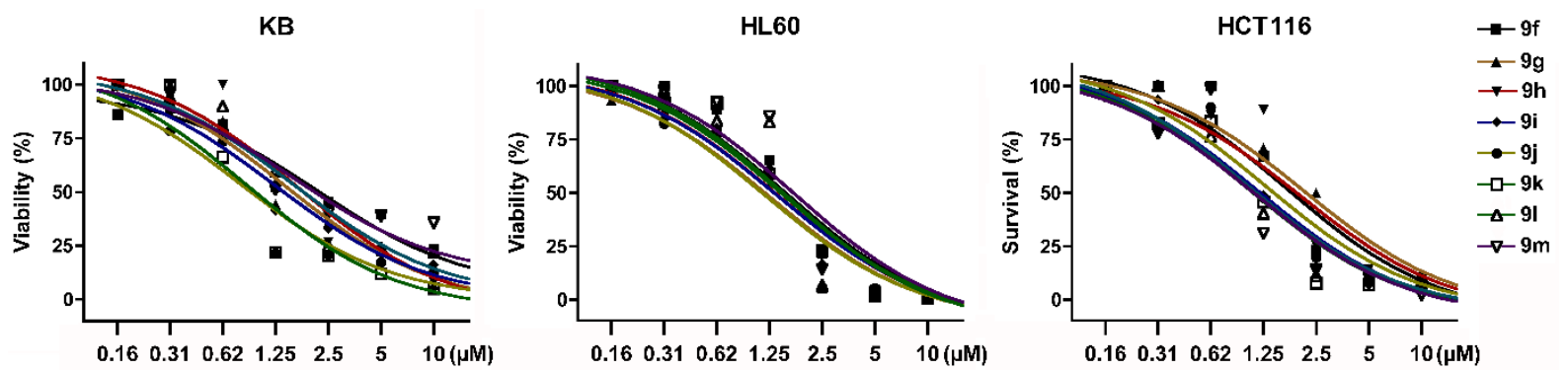

Fig. (1). Effects of iminobenzimidazoles 9f-m on the viability of KB, HL60 and HCT116 human cancer cells (average of two independent experiments). 
Table 2. Cytotoxicity in KB, HL60 and HCT116 human cancer cells $\left(\mathrm{IC}_{50}, \mu \mathrm{M}\right){ }^{[a]}$<smiles>[X][M]n1c(=N)n(CC(O)COc2ccc(Cl)cc2)c2ccccc21</smiles>

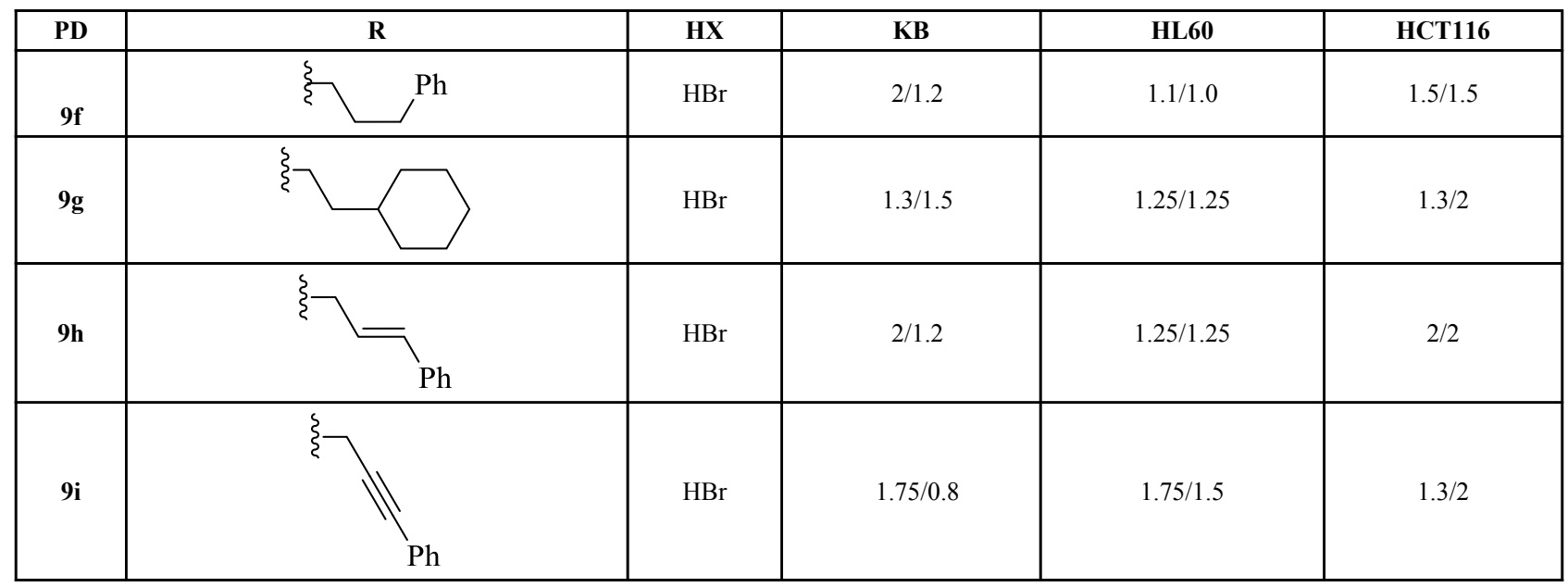

[a] Experiments were performed in duplicate.

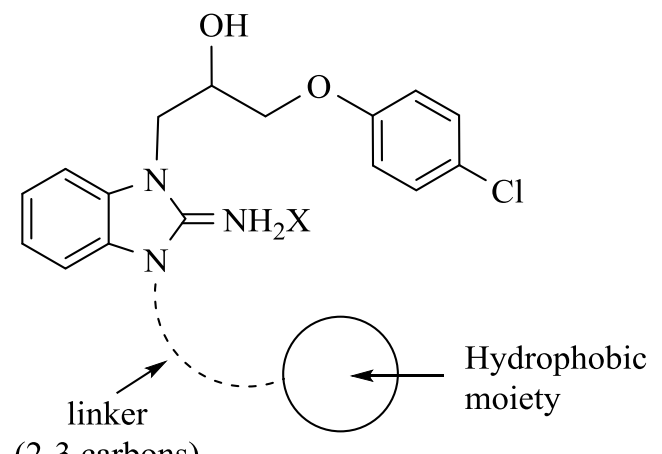

(2-3 carbons)

Fig. (2). Summary of the structural requirement of iminobenzimidazoles for their cytotoxicity.

\section{CONCLUSION}

The research and development of a novel type of drugs are of great importance to improve the treatment of cancers. In this context, we describe herein the discovery and optimization of the first-in-class series of cytotoxic agents. The SAR studies demonstrated the requirement for a hydrophobic moiety. These results are highly encouraging, and SAR studies to examine structural variations of the iminobenzimidazole and 3-(4-chlorophenoxy)-2-hydroxypropyl moieties are underway.

\section{CONSENT FOR PUBLICATION}

Not applicable.

\section{CONFLICT OF INTEREST}

The authors declare no conflict of interest, financial or otherwise. 


\section{ACKNOWLEDGEMENTS}

This work is published within the LABEX ANR-10-LABX-0034_Medalis and received financial support from the French government managed by ANR under "Programme d'investissement d'avenir". NC received a fellowship from Frères Mentouri University-Constantine 1.

\section{REFERENCES}

[1] Malka-Mahieu, H.; Newman, M.; Désaubry, L.; Robert, C.; Vagner, S. The eIF4F translation initiation complex- New opportunities for cancer treatment. Clin. Cancer Res., 2017, 23(1), 21-25.

[http://dx.doi.org/10.1158/1078-0432.CCR-14-2362] [PMID: 27789529]

[2] Basmadjian, C.; Thuaud, F.; Ribeiro, N.; Désaubry, L. Flavaglines: Potent anticancer drugs that target prohibitins and the helicase eIF4A. Future Med. Chem., 2013, 5(18), 2185-2197. [http://dx.doi.org/10.4155/fmc.13.177] [PMID: 24261894]

[3] Boussemart, L.; Malka-Mahieu, H.; Girault, I.; Allard, D.; Hemmingsson, O.; Tomasic, G.; Thomas, M.; Basmadjian, C.; Ribeiro, N.; Thuaud, F.; Mateus, C.; Routier, E.; Kamsu-Kom, N.; Agoussi, S.; Eggermont, A.M.; Désaubry, L.; Robert, C.; Vagner, S. eIF4F is a nexus of resistance to anti-BRAF and anti-MEK cancer therapies. Nature, 2014, 513(7516), 105-109. [http://dx.doi.org/10.1038/nature13572] [PMID: 25079330]

[4] Tabet, R.; Schaeffer, L.; Freyermuth, F.; Jambeau, M.; Workman, M.; Lee, C-Z.; Lin, C-C.; Jiang, J.; Jansen-West, K.; Abou-Hamdan, H.; Désaubry, L.; Gendron, T.; Petrucelli, L.; Martin, F.; Lagier-Tourenne, C. CUG initiation and frameshifting enable production of dipeptide repeat proteins from ALS/FTD C9ORF72 transcripts. Nat. Commun., 2018, 9(1), 152. [http://dx.doi.org/10.1038/s41467-017-02643-5] [PMID: 29323119]

[5] Malka-Mahieu, H.; Girault, I.; Rubington, M.; Leriche, M.; Welsch, C.; Kamsu-Kom, N.; Zhao, Q.; Désaubry, L.; Vagner, S.; Robert, C. Synergistic effects of eIF4A and MEK inhibitors on proliferation of NRAS-mutant melanoma cell lines. Cell Cycle, 2016, 15(18), $2405-2409$. [http://dx.doi.org/10.1080/15384101.2016.1208862] [PMID: 27533468]

[6] Wang, S.; Darini, C.; Désaubry, L.; Koromilas, A.E. Susceptibility to pharmacological inhibition of translation initiation factor eIF4A. Mol. Cancer Ther., 2016, 15(12), 3055-3063.

[http://dx.doi.org/10.1158/1535-7163.MCT-16-0416] [PMID: 27913706]

[7] Drasner, K.; Weber, K.T.U.S. U.S. Guanidine compounds as anesthetics and for treatment of nervous system disorders. Patent 7868028 B2, 2011.

[8] Yanagisawa, M. Preparation of iminobenzimidazole derivatives for use as type-2 orexin receptor agonists. U.S. Patent 20100150840 A1, 2010 .

[9] Raphemot, R.; Rouhier, M.F.; Hopkins, C.R.; Gogliotti, R.D.; Lovell, K.M.; Hine, R.M.; Ghosalkar, D.; Longo, A.; Beyenbach, K.W.; Denton, J.S.; Piermarini, P.M. Eliciting renal failure in mosquitoes with a small-molecule inhibitor of inward-rectifying potassium channels. PLoS One, 2013, 8(5), e64905. [http://dx.doi.org/10.1371/journal.pone.0064905] [PMID: 23734226]

[10] Rubin, H.; Reitz, A.; Selwood, T.; Yano, T.; Wrobel, J.; Loughran, H.M.; Weaver, D.; Costanzo, M.J. Preparation of substituted benzimidazoles as anti-infective compounds and methods using same., U.S. Patent 201462078726, 2014.

[11] Wolpaw, A.J.; Shimada, K.; Skouta, R.; Welsch, M.E.; Akavia, U.D.; Pe’er, D.; Shaik, F.; Bulinski, J.C.; Stockwell, B.R. Modulatory profiling identifies mechanisms of small molecule-induced cell death. Proc. Natl. Acad. Sci. USA, 2011, 108(39), E771-E780. [http://dx.doi.org/10.1073/pnas.1106149108] [PMID: 21896738]

\section{(C) 2018 Chouha et al.}

This is an open access article distributed under the terms of the Creative Commons Attribution 4.0 International Public License (CC-BY 4.0), a copy of which is available at: https://creativecommons.org/licenses/by/4.0/legalcode. This license permits unrestricted use, distribution, and reproduction in any medium, provided the original author and source are credited. 